\title{
Homogeneity of a Distance-Regular Graph Which Supports a Spin Model
}

\author{
BRIAN CURTIN \\ curtin@math.usf.edu \\ Department of Mathematics, University of South Florida, 4202 E. Fowler Ave. PHY 114, Tampa, FL 33647, USA \\ KAZUMASA NOMURA \\ nomura@tmd.ac.jp \\ College of Liberal Arts and Sciences, Tokyo Medical and Dental University, Kohnodai, Ichikawa, 272-0827, Japan
}

Received July 16, 2002; Revised March 25, 2003

\begin{abstract}
A spin model is a square matrix that encodes the basic data for a statistical mechanical construction of link invariants due to V.F.R. Jones. Every spin model $W$ is contained in a canonical Bose-Mesner algebra $\mathcal{N}(W)$. In this paper we study the distance-regular graphs $\Gamma$ whose Bose-Mesner algebra $\mathcal{M}$ satisfies $W \in \mathcal{M} \subseteq \mathcal{N}(W)$. Suppose $W$ has at least three distinct entries. We show that $\Gamma$ is 1 -homogeneous and that the first and the last subconstituents of $\Gamma$ are strongly regular and distance-regular, respectively.
\end{abstract}

Keywords: distance-regular graph, 1-homogeneous, spin model

\section{Introduction}

A spin model is a square matrix that encodes the basic data for a statistical mechanical construction of link invariants due to V.F.R. Jones [22]. Every spin model $W$ is contained in a canonical Bose-Mesner algebra $\mathcal{N}(W)$ [21, 37]. In many instances [1, 2, 19, 22, 33] there is a distance-regular graph whose Bose-Mesner algebra $\mathcal{M}$ satisfies $W \in \mathcal{M} \subseteq \mathcal{N}(W)$ we say that such a distance-regular graph supports the spin model $W$. These facts prompted the authors to study the distance-regular graphs which support a spin model $[10,12,14]$. The purpose of this paper is to further describe the combinatorial structure of these graphs.

Throughout this paper, all graphs will be finite without loops or multiple edges with pathlength distance function $\partial$. The $i$ th subconstituent of a graph $\Gamma$ with respect to a vertex $u$ is the set of all vertices at distance $i$ from $u$, and it is denoted by $\Gamma_{i}(u)$. When $\Gamma$ is connected, the number $d=\max \{\partial(x, y) \mid x, y$ are vertices of $\Gamma\}$ is called the diameter of $\Gamma$.

A connected graph $\Gamma$ with diameter $d$ is said to be distance-regular whenever there are integers $c_{i}, a_{i}, b_{i}(0 \leq i \leq d)$ such that for any vertices $u, v$ at distance $i=\partial(u, v)$, $\left|\Gamma_{i-1}(u) \cap \Gamma_{1}(v)\right|=c_{i},\left|\Gamma_{i}(u) \cap \Gamma_{1}(v)\right|=a_{i}$, and $\left|\Gamma_{i+1}(u) \cap \Gamma_{1}(v)\right|=b_{i}$. The integers $c_{i}$, $a_{i}, b_{i}$ are called the intersection numbers of $\Gamma$.

A connected graph $\Gamma$ is said to be $t$-homogeneous whenever for all vertices $u, v, w$ with $\partial(u, v)=t, \partial(u, w)=r, \partial(v, w)=s$, the number $\left|\Gamma_{i}(u) \cap \Gamma_{j}(v) \cap \Gamma_{1}(w)\right|$ is independent of the choice of $u, v, w$, depending only on $r, s, i, j$.

A graph is said to be strongly regular with parameters $(v, k, \lambda, \mu)$ when it has $v$ many vertices and for all vertices $u, v$, the number of common neighbors of $u$ and $v$ is $k$ if $u=v$, 
$\lambda$ if $u$ and $v$ are adjacent, and $\mu$ otherwise. A strongly regular graph is said to be trivial if $\mu=0$. A nontrivial strongly regular graph is simply a distance-regular graph with diameter 2 , and a trivial strongly regular graph is a disjoint union of cliques of the same size (see [6, Section 1.1]).

Theorem 1.1 Let $\Gamma$ denote a distance-regular graph with diameter $d \geq 1$. Suppose $\Gamma$ supports a spin model with at least three distinct entries. Pick any vertex $u$. Then

(i) The induced graph on $\Gamma_{1}(u)$ is strongly regular.

(ii) The induced graph on $\Gamma_{d}(u)$ is distance-regular.

(iii) $\Gamma$ is 1-homogeneous.

More complete statements of these results are given in Section 4, including formulas for the additional structure constants.

If $\Gamma$ is a nontrivial strongly regular graph which supports a spin model, then parts (i) and (ii) imply that $\Gamma$ is a strongly regular graph with strongly regular subconstituents, a fact shown in [19] (see also [17]). Such graphs were studied in [7]. The distance-regular graphs having all subconstituents strongly regular were characterized in [29]. Recently Caughman [8] showed that the $d$ th subconstituent of a Q-polynomial bipartite distance-regular graph $\Gamma$ is distance-regular, where two vertices in this graph are adjacent if they have distance 2 in $\Gamma$. Thus the induced graph on the last subconstituent of the halved graph of $\Gamma$ is distance-regular.

There are distance-regular graphs which satisfy conditions (i)-(iii) of Theorem 1.1 but do not support a spin model. One such example is the Hermitean dual polar space graph (see [6, Section 9.4]). In this case the induced graph on the last subconstituent is Hermitean forms graph (see [6, Section 9.5C]). The Hermitean dual polar space graphs are Q-polynomial with classical parameters. Comparing their parameterization to that of [12] shows that they cannot support a spin model.

The $t$-homogeneous property was first introduced in [32]. The 1-homogeneous graphs has since been studied extensively [25-28]. Bipartite and almost bipartite distance-regular graphs which support a spin model are trivially 1-homogeneous. In addition, they have the related 2-homogenous property [34-36]. The 2-homogeneous bipartite distance-regular graphs have been studied extensively by the authors and others in $[9,11,13,23,34,39]$. The 2-homogeneous almost bipartite distance-regular graphs have been studied in [36].

A connected graph $\Gamma$ is said to be triply regular when for all vertices $u, v, w$ and all $h, i, j$, the number $\left|\Gamma_{h}(u) \cap \Gamma_{i}(v) \cap \Gamma_{j}(w)\right|$ is independent of $u, v, w$, depending only on $h, i, j$ and the mutual distances between $u, v, w$. Triple regularity is stronger than conditions (i)-(iii) of Theorem 1.1. Triply regular graphs related to spin models were studied in [20].

The condition that a spin model has at least three distinct entries implies that any distanceregular graph which supports it has diameter at least 2 . The complete graph is the only distance-regular graph with diameter 1 , and it does in fact support a spin model with just two distinct entries. The complete graph trivially has the properties of Theorem 1.1. The link invariant associated with this spin model is the Jones polynomial [22]. There could, however, conceivably be other spin models with just two distinct entries. 


\section{Background}

Distance-regular graphs and spin models are related through Bose-Mesner algebras. We very briefly recall some general facts and establish some notation that we shall need, along with some references to further discussion. We shall recall other, more specialized results as necessary in the paper.

Fix a finite nonempty set $X$. Let $\operatorname{Mat}_{X}(\mathbb{C})$ denote the $\mathbb{C}$-algebra of complex matrices whose rows and columns are indexed by $X$. Let $\mathbb{C}^{X}$ denote the vector space of column tuples whose entries are indexed by $X$. Observe that $\operatorname{Mat}_{X}(\mathbb{C})$ acts on $\mathbb{C}^{X}$ by left multiplication. For $A \in M a t_{X}(\mathbb{C})$ and for $a, b \in X$, let $A(a, b)$ denote the $(a, b)$-entry of $A$. For $A, B \in$ $\operatorname{Mat}_{X}(\mathbb{C})$, let $A \circ B$ denote the Hadamard (entry-wise) product of $A$ and $B:(A \circ B)(x, y)=$ $A(x, y) B(x, y)$. The transpose of $A \in \operatorname{Mat}_{X}(\mathbb{C})$ is denoted by ${ }^{\mathrm{t}} A$.

Definition 2.1 A Bose-Mesner algebra on $X$ is a commutative subalgebra $\mathcal{M}$ of $\operatorname{Mat}_{X}(\mathbb{C})$, which is closed under the Hadamard product, which is closed under transposition, and which contains the identity matrix $I$ and the all 1's matrix $J$.

For more on Bose-Mesner algebras see [5, 6]. We use the following facts.

Lemma 2.2 Let $\mathcal{M}$ denote a $(d+1)$-dimensional Bose-Mesner algebra on $X$.

(i) $\mathcal{M}$ has a unique basis $\left\{A_{i}\right\}_{i=0}^{d}$, called the basis of Hadamard idempotents, such that $A_{0}=I, A_{i} \circ A_{j}=\delta_{i j} A_{i}(0 \leq i, j \leq d)$, and $\sum_{i=0}^{d} A_{i}=J$, where $\delta_{i j}$ is the Kronecker symbol.

(ii) $\mathcal{M}$ has a unique basis $\left\{E_{i}\right\}_{i=0}^{d}$, called the basis of primitive idempotents, such that $E_{0}=|X|^{-1} J, E_{i} E_{j}=\delta_{i j} E_{i}(0 \leq i, j \leq d)$, and $\sum_{i=0}^{d} E_{i}=I$.

Definition 2.3 Let $\mathcal{M}$ denote a Bose-Mesner algebra on $X$. A formal duality of $\mathcal{M}$ is a linear endomorphism $\Psi$ of $\mathcal{M}$ satisfying $\Psi(A B)=\Psi(A) \circ \Psi(B), \Psi(A \circ B)=$ $|X|^{-1} \Psi(A) \Psi(B)$, and $\Psi(\Psi(A))=|X|^{\mathrm{t}} A$ for all $A, B \in \mathcal{M}$.

For more on formal dualities see $[15,31]$. We use the following fact.

Lemma 2.4 Let $\mathcal{M}$ denote a $(d+1)$-dimensional Bose-Mesner algebra on X. Suppose that $\Psi$ is a formal duality of $\mathcal{M}$. Then there exist orderings $A_{0}, A_{1}, \ldots, A_{d}$ and $E_{0}, E_{1}, \ldots, E_{d}$ of the Hadamard and primitive idempotents such that $\Psi\left(E_{i}\right)=A_{i}, \Psi\left(A_{i}\right)=|X|^{\mathrm{t}} E_{i}(0 \leq$ $i \leq d$ ). Such orderings are called standard with respect to $\Psi$.

We now recall from $[5,6]$ some facts about the Bose-Mesner algebra of a distanceregular graph. Let $\Gamma$ denote a distance-regular graph with diameter $d$ and vertex set $X$. It is known that there are integers $p_{i, j}^{h}(0 \leq h, i, j \leq d)$ such that, for all $u, v \in X$ at distance $h=\partial(u, v)$,

$$
\left|\Gamma_{i}(u) \cap \Gamma_{j}(v)\right|=p_{i, j}^{h}
$$


Observe that $c_{0}=0, c_{i}=p_{1, i-1}^{i}(1 \leq i \leq d), a_{i}=p_{1, i}^{i}(0 \leq i \leq d), b_{i}=p_{1, i+1}^{i}$ $(0 \leq i \leq d-1)$, and $b_{d}=0$. In addition, we write $k_{i}=p_{i, i}^{0}(0 \leq i \leq d)$. For later reference we recall three formulas from [6, p. 134]:

$$
\begin{aligned}
p_{i, i}^{1} & =\frac{k_{i} a_{i}}{k_{1}}(0 \leq i \leq d), \quad p_{i, i+1}^{1}=\frac{k_{i} b_{i}}{k_{1}}(0 \leq i \leq d-1), \\
p_{i, i-1}^{1} & =\frac{k_{i} c_{i}}{k_{1}}(1 \leq i \leq d) .
\end{aligned}
$$

Recall that a distance-regular graph is bipartite when $a_{i}=0(0 \leq i \leq d)$ and almost bipartite when $a_{i}=0(0 \leq i \leq d-1), a_{d} \neq 0$.

Definition 2.5 Let $\Gamma$ denote a distance-regular graph with diameter $d$. For each integer $i(0 \leq i \leq d)$, the $i$ th distance matrix of $\Gamma$ is the matrix $A_{i} \in \operatorname{Mat}_{X}(\mathbb{C})$ with $(x, y)$-entry $A_{i}(x, y)=1$ if $\partial(x, y)=i$ and 0 otherwise. The matrix $A=A_{1}$ is the adjacency matrix of $\Gamma$.

Lemma 2.6 Let $\Gamma$ denote a distance-regular graph with diameter d, let $A_{0}, A=A_{1}, \ldots$, $A_{d}$ denote the distance-matrices of $\Gamma$, and let $\mathcal{M}$ denote the linear span of $\left\{A_{i}\right\}_{i=0}^{d}$.

(i) $A_{i}$ is a polynomial of degree exactly $i$ in $A(0 \leq i \leq d)$.

(ii) $A_{i} A_{j}=\sum_{h=0}^{d} p_{i, j}^{h} A_{h}(0 \leq i, j \leq d)$.

(iii) $\mathcal{M}$ is a Bose-Mesner algebra, called the Bose-Mesner algebra of $\Gamma$.

(iv) $\left\{A_{i}\right\}_{i=0}^{d}$ is the basis of Hadamard idempotents of $\mathcal{M}$.

(v) Every matrix in $\mathcal{M}$ is symmetric.

(vi) A has exactly $d+1$ distinct eigenvalues.

(vii) A has spectral decomposition $A=\sum_{i=0}^{d} \theta_{i} E_{i}$, where $E_{0}, E_{1}, \ldots, E_{d}$ are the orthogonal projections onto the maximal eigenspaces of $A$ and $\theta_{0}, \theta_{1}, \ldots, \theta_{d}$ are the corresponding eigenvalues.

(viii) $\left\{E_{i}\right\}_{i=0}^{d}$ is the basis of primitive idempotents of $\mathcal{M}$.

(ix) ${ }^{\mathfrak{t}} E_{i}=\bar{E}_{i}=E_{i}(0 \leq i \leq d)$.

Definition 2.7 Let $\Gamma$ denote a distance-regular graph with diameter $d \geq 1$. An ordering $E_{0}, E_{1}, \ldots, E_{d}$ of the primitive idempotents of $\Gamma$ is called a $Q$-polynomial ordering whenever for all $i(0 \leq i \leq d), E_{i}$ is a polynomial of exactly degree $i$ in $E_{1}$ (with respect to the Hadamard product). Let $E$ denote a primitive idempotent. Then $\Gamma$ is said to be $Q$-polynomial with respect to $E$ when there exists a Q-polynomial ordering of the primitive idempotents with $E=E_{1}$.

We now recall some facts about spin models and the Bose-Mesner algebras which support them. Symmetric spin models were first introduced by Jones in [22] and later generalized in $[3,30]$. It is symmetric spin models which arise in this paper. The following facts relating spin models to Bose-Mesner algebras appear in [21, 37]. 
Definition 2.8 A spin model on $X$ is a symmetric matrix $W \in \operatorname{Mat}_{X}(\mathbb{C})$ with non-zero entries which satisfy the following equations for all $a, b, c \in X$ :

$$
\begin{aligned}
\sum_{x \in X} W(x, b) W(x, c)^{-1} & =|X| \delta_{b c}, \\
\sum_{x \in X} W(x, a) W(x, b) W(x, c)^{-1} & =\sqrt{|X|} W(a, b) W(a, c)^{-1} W(c, b)^{-1} .
\end{aligned}
$$

Let $W$ denote a spin model on $X$. For all $b, c \in X$, define $\mathbf{u}_{b c} \in \mathbb{C}^{X}$ to have $x$-entry $\mathbf{u}_{b c}(x)=W(x, b) W(x, c)^{-1}(x \in X)$. Define $\mathcal{N}(W)$ to be the set of all matrices $A \in$ $\operatorname{Mat}_{X}(\mathbb{C})$ such that for all $b, c \in X$, the vector $\mathbf{u}_{b c}$ is an eigenvector of $A$. For $A \in \mathcal{N}(W)$, let $\Psi(A) \in \operatorname{Mat}_{X}(\mathbb{C})$ be defined by $A \mathbf{u}_{b c}=(\Psi(A))(b, c) \mathbf{u}_{b c}$ for all $b, c \in X$.

Theorem $2.9[21,37] \quad$ Let $W$ denote a spin model on $X$. Then with the notation of Definition 2.8, the following hold.

(i) $W \in \mathcal{N}(W)$.

(ii) $\mathcal{N}(W)$ is a Bose-Mesner algebra.

(iii) $\Psi$ is a formal duality of $\mathcal{N}(W)$.

Definition 2.10 Let $\mathcal{M}$ denote a Bose-Mesner algebra on $X$ and let $W$ denote a spin model on $X . \mathcal{M}$ is said to support $W$ whenever $W \in \mathcal{M} \subseteq \mathcal{N}(W)$.

Bose-Mesner algebras which support a spin model have been studied by the authors in $[10,12,14]$. In addition to the following result, we shall recall several facts in the body of the paper about distance-regular graphs whose Bose-Mesner algebra supports a spin model.

Lemma 2.11 [10,12] Let $\mathcal{M}$ denote a Bose-Mesner algebra on $X$ which supports a spin model $W$. Then the map $\Psi$ of Definition 2.8 satisfies $\Psi(\mathcal{M})=\mathcal{M}$, and $\Psi$ induces a formal duality of $\mathcal{M}$.

\section{Structure}

All three parts of Theorem 1.1 involve counting the number of vertices in certain configurations relative to three vertices. In each case one of the distances is one, so they can be proved by showing that the numbers $\left|\Gamma_{i}(u) \cap \Gamma_{j}(v) \cap \Gamma_{1}(w)\right|$ are independent of $u, v, w$ for certain configurations of these three vertices. In this section we prove the structural results for Theorem 1.1, although we postpone the proof of Theorem 1.1 until the next section.

Let $\Gamma$ denote a distance-regular graph with diameter $d \geq 2$ and vertex set $X$, and let $\mathcal{M}$ denote the Bose-Mesner algebra of $\Gamma$. Let $W$ denote a spin model on $X$, and suppose that $\Gamma$ supports $W$. Let $A_{0}, A_{1}, \ldots, A_{d}$ denote the distance-matrices of $\Gamma$. Since $W \in \mathcal{M}$, we may write

$$
W=\sum_{i=0}^{d} t_{i} A_{i} .
$$


Observe that $t_{0}, \ldots, t_{d}$ are the entries of $W$. Let $\Psi$ denote the formal duality of $\mathcal{M}$ from Definition 2.8 and Lemma 2.11. Let $E_{0}, E_{1}, \ldots, E_{d}$ denote the primitive idempotents of $\mathcal{M}$ in the standard ordering with respect to $\Psi$. Let $\theta_{0}, \theta_{1}, \ldots, \theta_{d}$ denote the associated eigenvalues of the adjacency matrix $A$ of $\Gamma$.

For $u, v \in X$, we write

$$
\mathrm{D}_{j}^{i}(u, v)=\Gamma_{i}(u) \cap \Gamma_{j}(v)
$$

The number of edges from a vertex $u$ into a subset $Y \subset X$ is denoted by $e(u, Y)$.

Lemma 3.1 Let $u, v, w \in X$ be vertices at distance $h=\partial(u, v), r=\partial(u, w)$, and $s=\partial(v, w)$. Then

(i) If one of $h, i, j$ is greater than the sum of the other two, then $\mathrm{D}_{j}^{i}(u, v)=\emptyset$. In particular, $e\left(w, \mathrm{D}_{j}^{i}(u, v)\right)=0$ in this case.

(ii) $e\left(w, \mathrm{D}_{j}^{i}(u, v)\right)=0$ unless $r-1 \leq i \leq r+1$ and $s-1 \leq j \leq s+1$.

Proof: Immediate from the triangle inequality for $\partial$.

We picture the nine possible vertex counts allowed by Lemma 3.1(ii) as follows.

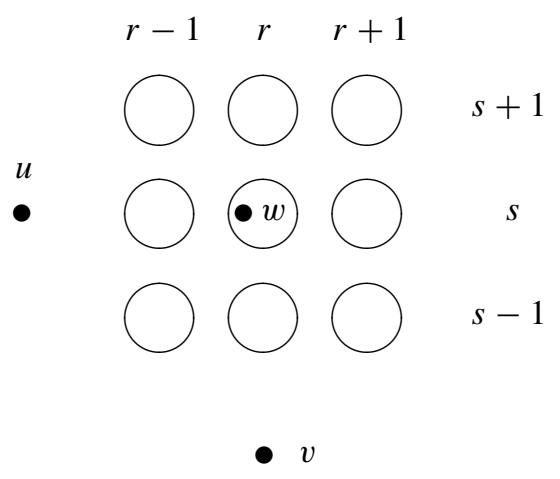

Lemma 3.2 Let $u, v \in X$ be vertices at distance $h=\partial(u, v)$. Then for all $r, s(0 \leq r, s \leq$ d) and for all $w \in \mathrm{D}_{s}^{r}(u, v)$,

$$
\begin{aligned}
& b_{r}=e\left(w, \mathrm{D}_{s-1}^{r+1}(u, v)\right)+e\left(w, \mathrm{D}_{s}^{r+1}(u, v)\right)+e\left(w, \mathrm{D}_{s+1}^{r+1}(u, v)\right), \\
& a_{r}=e\left(w, \mathrm{D}_{s-1}^{r}(u, v)\right)+e\left(w, \mathrm{D}_{s}^{r}(u, v)\right)+e\left(w, \mathrm{D}_{s+1}^{r}(u, v)\right), \\
& c_{r}=e\left(w, \mathrm{D}_{s-1}^{r-1}(u, v)\right)+e\left(w, \mathrm{D}_{s}^{r-1}(u, v)\right)+e\left(w, \mathrm{D}_{s+1}^{r-1}(u, v)\right), \\
& b_{s}=e\left(w, \mathrm{D}_{s+1}^{r-1}(u, v)\right)+e\left(w, \mathrm{D}_{s+1}^{r}(u, v)\right)+e\left(w, \mathrm{D}_{s+1}^{r+1}(u, v)\right), \\
& a_{s}=e\left(w, \mathrm{D}_{s}^{r-1}(u, v)\right)+e\left(w, \mathrm{D}_{s}^{r}(u, v)\right)+e\left(w, \mathrm{D}_{s}^{r+1}(u, v)\right), \\
& c_{s}=e\left(w, \mathrm{D}_{s-1}^{r-1}(u, v)\right)+e\left(w, \mathrm{D}_{s-1}^{r}(u, v)\right)+e\left(w, \mathrm{D}_{s-1}^{r+1}(u, v)\right) .
\end{aligned}
$$


Proof: Clear from the definition of the intersection numbers.

The equations of Lemma 3.2 are not independent - the sum of the first three is equal to the sum of the last three. Spin models give two more equations.

Lemma 3.3 [12, Lemma 4.2] Let $u, v \in X$ be vertices at distance $h=\partial(u, v)$. Then for all $r, s(0 \leq r, s \leq d)$ and for all $w \in \mathrm{D}_{s}^{r}(u, v)$,

$$
\begin{aligned}
& \sum_{i=r-1}^{r+1} \sum_{j=s-1}^{s+1} e\left(w, \mathrm{D}_{j}^{i}(u, v)\right) \frac{t_{i}}{t_{j}}=\theta_{h} \frac{t_{r}}{t_{s}}, \\
& \sum_{i=r-1}^{r+1} \sum_{j=s-1}^{s+1} e\left(w, \mathrm{D}_{j}^{i}(u, v)\right) \frac{t_{j}}{t_{i}}=\theta_{h} \frac{t_{s}}{t_{r}}
\end{aligned}
$$

Observe that (3) is obtained from (2) by replacing each $t_{\ell}$ with $t_{\ell}^{-1}$ for all $\ell$.

Lemma 3.4 [12, Corollaries 4.6 and 4.7]

(i) If $t_{1} \in\left\{t_{0},-t_{0}\right\}$, then $t_{r} \in\left\{t_{0},-t_{0}\right\}(0 \leq r \leq d)$.

(ii) If $t_{1} \notin\left\{t_{0},-t_{0}\right\}$, then $t_{r-1}, t_{r}, t_{r+1}$ are mutually distinct $(1 \leq r \leq d-1)$.

Observe that, from Lemma 3.4, if $W$ has at least three distinct entries then $t_{r-1}, t_{r}, t_{r+1}$ are mutually distinct $(1 \leq r \leq d-1)$.

Lemma 3.5 Suppose that $t_{1} \notin\left\{t_{0},-t_{0}\right\}$. Let $r$, s be integers $(0 \leq r, s \leq d)$ which satisfy at least one of the following conditions.

(i) $r+s=h$,

(ii) $|r-s|=h$,

(iii) $r=1$ or $s=1$,

(iv) $r=d$ or $s=d$.

Then, for all integers $i, j$ and for all vertices $u, v, w$ such that $\partial(u, v)=h$ and $w \in \mathrm{D}_{s}^{r}(u, v)$, the number $e\left(w, \mathrm{D}_{j}^{i}(u, v)\right)$ is independent of the choice of $u, v, w$, depending only on $h, r$, $s, i, j$.

Proof: $\quad$ Set $\mathrm{D}_{j}^{i}=\mathrm{D}_{j}^{i}(u, v)$ for all $i, j$.

(i) Suppose $r+s=h$. Observe that $\mathrm{D}_{s-1}^{r-1}=\mathrm{D}_{s}^{r-1}=\mathrm{D}_{s-1}^{r}=\emptyset$ by Lemma 3.1(i). Now pick any $w \in \mathrm{D}_{s}^{r}$ and set $e\left(w, \mathrm{D}_{s}^{r}\right)=\alpha$. Then by Lemma 3.2, $e\left(w, \mathrm{D}_{s+1}^{r-1}\right)=c_{r}, e\left(w, \mathrm{D}_{s-1}^{r+1}\right)=$ $c_{s}, e\left(w, \mathrm{D}_{s+1}^{r}\right)=a_{r}-\alpha, e\left(w, \mathrm{D}_{s}^{r+1}\right)=a_{s}-\alpha$, and $e\left(w, \mathrm{D}_{s+1}^{r+1}\right)=b_{r}-c_{s}-\left(a_{s}-\alpha\right)$. Thus it is enough to show that $\alpha$ does not depend on $u, v, w$. Substituting these expressions into (2) gives

$$
\begin{aligned}
\theta_{h} \frac{t_{r}}{t_{s}}= & \alpha \frac{t_{r}}{t_{s}}+c_{r} \frac{t_{r-1}}{t_{s+1}}+c_{s} \frac{t_{r+1}}{t_{s-1}}+\left(a_{r}-\alpha\right) \frac{t_{r}}{t_{s+1}} \\
& +\left(a_{s}-\alpha\right) \frac{t_{r+1}}{t_{s}}+\left(b_{r}-c_{s}-\left(a_{s}-\alpha\right)\right) \frac{t_{r+1}}{t_{s+1}} .
\end{aligned}
$$


The coefficient of $\alpha$ is

$$
\frac{t_{r}}{t_{s}}-\frac{t_{r}}{t_{s+1}}-\frac{t_{r+1}}{t_{s}}+\frac{t_{r+1}}{t_{s+1}}=\frac{\left(t_{r}-t_{r+1}\right)\left(t_{s+1}-t_{s}\right)}{t_{s} t_{s+1}},
$$

which is not zero by Lemma 3.4. Solving (4) for $\alpha$ shows that $\alpha$ is independent of $u, v, w$.

(ii) Suppose $r-s=h$. (The case $s-r=h$ is similar). Observe that $\mathrm{D}_{s-1}^{r}=\mathrm{D}_{s-1}^{r+1}=$ $\mathrm{D}_{s}^{r+1}=\emptyset$ by Lemma 3.1(i). Now pick any $w \in \mathrm{D}_{s}^{r}$ and set $e\left(w, \mathrm{D}_{s}^{r}\right)=\alpha$. Then by Lemma 3.2, $e\left(w, \mathrm{D}_{s-1}^{r-1}\right)=c_{s}, e\left(w, \mathrm{D}_{s}^{r-1}\right)=a_{s}-\alpha, e\left(w, \mathrm{D}_{s+1}^{r}\right)=a_{r}-\alpha, e\left(w, \mathrm{D}_{s+1}^{r+1}\right)=b_{r}$, $e\left(w, \mathrm{D}_{s+1}^{r-1}\right)=c_{r}-c_{s}-\left(a_{s}-\alpha\right)$. Proceeding as in (i) to substitute these expressions into (2), we find that the coefficient of $\alpha$ is

$$
\frac{t_{r}}{t_{s}}-\frac{t_{r-1}}{t_{s}}-\frac{t_{r}}{t_{s+1}}+\frac{t_{r-1}}{t_{s+1}}=\frac{\left(t_{r}-t_{r-1}\right)\left(t_{s+1}-t_{s}\right)}{t_{s} t_{s+1}} \neq 0
$$

Thus $\alpha$ is independent of the choice of $u, v, w$.

(iii) Suppose $r=1$. (The case $s=1$ is similar). Then $s \in\{h-1, h, h+1\}$ by Lemma 3.1(ii). The case $s=h-1$ is covered by (i), and the case $s=h+1$ is covered by (ii). So we assume $s=h$. Observe that $\mathrm{D}_{s-1}^{r-1}=\mathrm{D}_{s+1}^{r-1}=\emptyset$ by Lemma 3.1(i), and $\mathrm{D}_{s}^{r-1}=\{u\}$. Pick any $w \in \mathrm{D}_{s}^{r}$ and set $e\left(w, \mathrm{D}_{s}^{r}\right)=\alpha, e\left(w, \mathrm{D}_{s-1}^{r}\right)=\gamma$. Then by Lemma 3.2, $e\left(w, \mathrm{D}_{s}^{r-1}\right)=1, e\left(w, \mathrm{D}_{s}^{r+1}\right)=a_{s}-\alpha-1, e\left(w, \mathrm{D}_{s+1}^{r}\right)=a_{r}-\alpha-\gamma, e\left(w, \mathrm{D}_{s-1}^{r+1}\right)=c_{s}-\gamma$, $e\left(w, \mathrm{D}_{s+1}^{r+1}\right)=b_{s}-\left(a_{r}-\alpha-\gamma\right)$. Substituting these expressions into (2) and (3) gives, respectively,

$$
\begin{aligned}
\theta_{h} \frac{t_{r}}{t_{s}}= & \alpha \frac{t_{r}}{t_{s}}+\gamma \frac{t_{r}}{t_{s-1}}+\frac{t_{r-1}}{t_{s}}+\left(a_{s}-\alpha-1\right) \frac{t_{r+1}}{t_{s}}+\left(a_{r}-\alpha-\gamma\right) \frac{t_{r}}{t_{s+1}} \\
& +\left(c_{s}-\gamma\right) \frac{t_{r+1}}{t_{s-1}}+\left(b_{s}-\left(a_{r}-\alpha-\gamma\right)\right) \frac{t_{r+1}}{t_{s+1}}, \\
\theta_{h} \frac{t_{s}}{t_{r}}= & \alpha \frac{t_{s}}{t_{r}}+\gamma \frac{t_{s-1}}{t_{r}}+\frac{t_{s}}{t_{r-1}}+\left(a_{s}-\alpha-1\right) \frac{t_{s}}{t_{r+1}}+\left(a_{r}-\alpha-\gamma\right) \frac{t_{s+1}}{t_{r}} \\
& +\left(c_{s}-\gamma\right) \frac{t_{s-1}}{t_{r+1}}+\left(b_{s}-\left(a_{r}-\alpha-\gamma\right)\right) \frac{t_{s+1}}{t_{r+1}} .
\end{aligned}
$$

We view the above two equations as a system of linear equations with unknowns $\alpha, \gamma$. The coefficient matrix of $\alpha$ and $\gamma$ is

$$
\left(\begin{array}{ll}
\frac{t_{r}}{t_{s}}-\frac{t_{r+1}}{t_{s}}-\frac{t_{r}}{t_{s+1}}+\frac{t_{r+1}}{t_{s+1}} & \frac{t_{r}}{t_{s-1}}-\frac{t_{r}}{t_{s+1}}-\frac{t_{r+1}}{t_{s-1}}+\frac{t_{r+1}}{t_{s+1}} \\
\frac{t_{s}}{t_{r}}-\frac{t_{s}}{t_{r+1}}-\frac{t_{s+1}}{t_{r}}+\frac{t_{s+1}}{t_{r+1}} & \frac{t_{s-1}}{t_{r}}-\frac{t_{s+1}}{t_{r}}-\frac{t_{s-1}}{t_{r+1}}+\frac{t_{s+1}}{t_{r+1}}
\end{array}\right),
$$

which has determinant

$$
\frac{\left(t_{r+1}-t_{r}\right)^{2}\left(t_{s-1}-t_{s}\right)\left(t_{s+1}-t_{s}\right)\left(t_{s+1}-t_{s-1}\right)}{t_{r} t_{r+1} t_{s-1} t_{s} t_{s+1}} \neq 0 .
$$


Thus $\alpha, \gamma$ are independent of the choice of $u, v, w$.

(iv) Suppose $r=d$. (The case $s=d$ is similar). Observe that $\mathrm{D}_{s-1}^{r+1}=\mathrm{D}_{s}^{r+1}=\mathrm{D}_{s+1}^{r+1}=\emptyset$ by Lemma 3.1(i). Now pick any $w \in \mathrm{D}_{s}^{r}$ and set $e\left(w, \mathrm{D}_{s}^{r}\right)=\alpha, e\left(w, \mathrm{D}_{s-1}^{r}\right)=\gamma$. Then by Lemma 3.2,e $e\left(w, \mathrm{D}_{s-1}^{r-1}\right)=c_{s}-\gamma, e\left(w, \mathrm{D}_{s}^{r-1}\right)=a_{s}-\alpha, e\left(w, \mathrm{D}_{s+1}^{r}\right)=a_{r}-\alpha-\gamma$, $e\left(w, \mathrm{D}_{s+1}^{r-1}\right)=b_{s}-\left(a_{r}-\alpha-\gamma\right)$. Proceeding as in (iii) to substitute these expressions into (2) and (3) we find the coefficient matrix of $\alpha$ and $\gamma$ to be

$$
\left(\begin{array}{ll}
\frac{t_{r}}{t_{s}}-\frac{t_{r-1}}{t_{s}}-\frac{t_{r}}{t_{r}}+\frac{t_{r-1}}{t_{s+1}} & \frac{t_{r}}{t_{s-1}}-\frac{t_{r-1}}{t_{s-1}}-\frac{t_{r}}{t_{s+1}}+\frac{t_{r-1}}{t_{s+1}} \\
\frac{t_{s}}{t_{r}}-\frac{t_{s}}{t_{r-1}}-\frac{t_{s+1}}{t_{r}}+\frac{t_{s+1}}{t_{r-1}} & \frac{t_{s-1}}{t_{r}}-\frac{t_{s-1}}{t_{r-1}}-\frac{t_{s+1}}{t_{r}}+\frac{t_{s+1}}{t_{r-1}}
\end{array}\right) .
$$

This matrix has determinant

$$
\frac{\left(t_{r-1}-t_{r}\right)^{2}\left(t_{s-1}-t_{s}\right)\left(t_{s}-t_{s+1}\right)\left(t_{s-1}-t_{s+1}\right)}{t_{r-1} t_{r} t_{s-1} t_{s} t_{s+1}} \neq 0 .
$$

Thus $\alpha, \gamma$ are independent of the choice of $u, v, w$.

Observe that if $d=2$, then the above implies that $\Gamma$ is 1-homogeneous. For larger diameter, we still need to treat the case $h=1$ and $r=s$. In this case (2) and (3) are dependent, so we need one more equation. We draw it from Terwilliger's balanced sets condition [38]. To apply this result, we need a few preliminary results and some notation. These facts are generally well-known, but we verify that they do indeed apply to the situation described at the beginning of the section. We recall that in this situation we have fixed a distance-regular graph $\Gamma$ with diameter $d \geq 2$ which supports a spin model $W$. The BoseMesner algebra $\mathcal{M}$ of $\Gamma$ has a formal duality $\Psi$ given as in Definition 2.8. We also write $A$ to denote the adjacency matrix of $\Gamma$ and $\left\{E_{i}\right\}_{i=0}^{d}$ to denote the basis of primitive idempotents of $\mathcal{M}$.

Lemma 3.6 The standard ordering $E_{0}, E_{1}, \ldots E_{d}$ for $\Psi$ is also a $Q$-polynomial ordering of the primitive idempotents.

Proof: Apply $\Psi$ to Lemma 2.6(i), and simplify with Definition 2.3.

Definition 3.7 Given a primitive idempotent $E$ of $\Gamma$, the scalars $\theta_{0}^{*}, \theta_{1}^{*}, \ldots, \theta_{d}^{*}$ such that $E=|X|^{-1} \sum_{i=0}^{d} \theta_{i}^{*} A_{i}$ are called the dual eigenvalues of $\Gamma$ associated with $E$.

Lemma 3.8 Let $E_{0}, E_{1}, \ldots, E_{d}$ denote the primitive idempotents of $\Gamma$ in the standard ordering for $\Psi$, and let $\theta_{0}, \theta_{1}, \ldots, \theta_{d}$ denote the corresponding eigenvalues of $A$. Let $\theta_{0}^{*}, \theta_{1}^{*}, \ldots, \theta_{d}^{*}$ denote the dual eigenvalues of $\Gamma$ associated with $E_{1}$. Then $\theta_{i}^{*}=\theta_{i}(0 \leq i \leq$ d).

Proof: On one hand, the spectral decomposition of $A$ is $A=\sum_{i=0}^{d} \theta_{i} E_{i}$. Applying $\Psi$ gives $|X| E_{1}=\sum_{i=0}^{d} \theta_{i} A_{i}$. On the other hand, $E_{1}=|X|^{-1} \sum_{i=0}^{d} \theta_{i}^{*} A_{i}$ by the definition of the dual eigenvalues. 
Definition 3.9 For all $u \in X$, let $\hat{u}$ denote the characteristic vector of $u$ in $\mathbb{C}^{X}: \hat{u}(x)=1$ if $x=u$, and 0 otherwise. For any subset $Y \subset X$, we write $\hat{Y}=\sum_{u \in Y} \hat{u}$.

The following lemma is well-known. We endow $\mathbb{C}^{X}$ with the Hermitian inner product $\langle$,$\rangle defined by \langle\mathbf{u}, \mathbf{v}\rangle=\mathbf{u} \overline{\mathbf{v}}\left(\mathbf{u}, \mathbf{v} \in \mathbb{C}^{X}\right)$.

Lemma 3.10 Let $E$ denote a primitive idempotent of $\Gamma$, and let $\theta_{0}^{*}, \theta_{1}^{*}, \ldots, \theta_{d}^{*}$ denote the dual eigenvalues of $\Gamma$ associated with $E$. Then for all $x, y \in X,\langle E \hat{x}, E \hat{y}\rangle=|X|^{-1} \theta_{h}^{*}$, where $h=\partial(x, y)$.

Proof: By Lemma 2.6(ix) ${ }^{\mathfrak{t}} E=E$ and $\bar{E}=E$. By the idempotent property, $E^{2}=E$. By Definition $3.7 E=|X|^{-1} \sum_{i=0}^{d} \theta_{i}^{*} A_{i}$. With this information we compute $\langle E \hat{x}, E \hat{y}\rangle=$ $\left\langle{ }^{t} \bar{E} E \hat{x}, \hat{y}\right\rangle=\langle E \hat{x}, \hat{y}\rangle=|X|^{-1} \sum_{i=0}^{d} \theta_{i}^{*}\left\langle A_{i} \hat{x}, \hat{y}\right\rangle=|X|^{-1} \theta_{h}^{*}$.

Theorem 3.11 (Terwilliger [38, Theorem 3.3(vii)]) Let $\Gamma$ denote a distance-regular graph with diameter $d \geq 3$. Let $E$ denote a primitive idempotent of $\Gamma$, and let $\theta_{0}^{*}, \theta_{1}^{*}, \ldots, \theta_{d}^{*}$ denote the dual eigenvalues of $\Gamma$ associated with $E$. Suppose $\Gamma$ is $Q$-polynomial with respect to $E$. Then $\theta_{\ell}^{*} \neq \theta_{0}^{*}(1 \leq \ell \leq d)$ and for all $i, j(1 \leq i, j \leq d)$ and for all $u, v \in X$

$$
\widehat{E \mathrm{D}_{j}^{i}}(u, v)-E \widehat{\mathrm{D}_{i}^{j}}(u, v)=p_{i, j}^{h} \frac{\theta_{i}^{*}-\theta_{j}^{*}}{\theta_{0}^{*}-\theta_{h}^{*}}(E \hat{u}-E \hat{v}),
$$

where $h=\partial(u, v)$.

Lemma 3.12 [12, Lemma 4.5] For all $r(1 \leq r \leq d)$,

$$
\frac{\frac{t_{r}}{t_{r-1}}-\frac{t_{r-1}}{t_{r}}}{\theta_{r-1}-\theta_{r}}=\frac{\frac{t_{1}}{t_{0}}-\frac{t_{0}}{t_{1}}}{\theta_{0}-\theta_{1}}
$$

Lemma 3.13 Assume that $t_{1} \notin\left\{t_{0},-t_{0}\right\}$. Then for all $r(2 \leq r \leq d-1)$ and for all vertices $u, v, w$ with $\partial(u, v)=1$ and $w \in \mathrm{D}_{r}^{r}(u, v)$, the number $e\left(w, \mathrm{D}_{j}^{i}(u, v)\right)$ is independent of the choice of $u, v, w$.

Proof: We may assume $d \geq 3$. Let $u, v \in X$ with $\partial(u, v)=1$, and write $\mathrm{D}_{j}^{i}=\mathrm{D}_{j}^{i}(u, v)$ for all $i, j$. Observe that $\mathrm{D}_{r+1}^{r-1}=\mathrm{D}_{r-1}^{r+1}=\emptyset$ by Lemma 3.1(i). Now pick any $w \in \mathrm{D}_{r}^{r}$ and set $e\left(w, \mathrm{D}_{r-1}^{r-1}\right)=\gamma, e\left(w, \mathrm{D}_{r+1}^{r+1}\right)=\beta$. Then by Lemma 3.2, $e\left(w, \mathrm{D}_{r}^{r-1}\right)=c_{r}-\gamma, e\left(w, \mathrm{D}_{r-1}^{r}\right)=$ $c_{r}-\gamma, e\left(w, \mathrm{D}_{r+1}^{r}\right)=b_{r}-\beta, e\left(w, \mathrm{D}_{r}^{r+1}\right)=b_{r}-\beta, e\left(w, \mathrm{D}_{r}^{r}\right)=a_{r}-\left(c_{r}-\gamma\right)-\left(b_{r}-\beta\right)$. Thus the result will follow once we have shown that $\beta$ and $\gamma$ do not depend upon $u, v, w$. Substituting these expressions into (2) gives

$$
\begin{aligned}
\theta_{1} \frac{t_{r}}{t_{r}}= & \gamma \frac{t_{r-1}}{t_{r-1}}+\beta \frac{t_{r+1}}{t_{r+1}}+\left(c_{r}-\gamma\right)\left(\frac{t_{r-1}}{t_{r}}+\frac{t_{r}}{t_{r-1}}\right) \\
& +\left(b_{r}-\beta\right)\left(\frac{t_{r}}{t_{r+1}}+\frac{t_{r+1}}{t_{r}}\right)+\left(a_{r}-\left(c_{r}-\gamma\right)-\left(b_{r}-\beta\right)\right) \frac{t_{r}}{t_{r}} .
\end{aligned}
$$


Thus

$$
\left(2-\frac{t_{r}}{t_{r+1}}-\frac{t_{r+1}}{t_{r}}\right) \beta+\left(2-\frac{t_{r-1}}{t_{r}}-\frac{t_{r}}{t_{r-1}}\right) \gamma=\zeta_{1}
$$

a constant which is independent of $u, v, w$.

Write $\tilde{\mathrm{D}}_{j}^{i}=\mathrm{D}_{j}^{i}(u, w)$ for all $i$ and $j$. By Theorem 3.11 and Lemma 3.8,

$$
a_{r} \frac{\theta_{1}-\theta_{r}}{\theta_{0}-\theta_{r}}(E \hat{u}-E \hat{w})=E \widehat{\tilde{\mathrm{D}}_{r}^{1}}-E \widehat{\widetilde{\mathrm{D}}_{1}^{r}}
$$

We derive a relation involving $\beta$ and $\gamma$ by computing the inner product of $E \hat{v}$ with the each side of (7) and using Lemma 3.10. To make this computation, observe that $\tilde{\mathrm{D}}_{1}^{r}=$ $\left(\tilde{\mathrm{D}}_{1}^{r} \cap \Gamma_{r-1}(v)\right) \cup\left(\tilde{\mathrm{D}}_{1}^{r} \cap \Gamma_{r}(v)\right) \cup\left(\tilde{\mathrm{D}}_{1}^{r} \cap \Gamma_{r+1}(v)\right)$ and that $\left|\tilde{\mathrm{D}}_{1}^{r} \cap \Gamma_{r-1}(v)\right|=e\left(w, \mathrm{D}_{r-1}^{r}\right)=$ $c_{r}-\gamma,\left|\tilde{\mathrm{D}}_{1}^{r} \cap \Gamma_{r}(v)\right|=e\left(w, \mathrm{D}_{r}^{r}\right)=a_{r}-\left(c_{r}-\gamma\right)-\left(a_{r}-\beta\right)$, and $\left|\tilde{\mathrm{D}}_{1}^{r} \cap \Gamma_{r+1}(v)\right|=$ $e\left(w, \mathrm{D}_{r+1}^{r}\right)=b_{r}-\beta$.

Also observe that $\alpha^{\prime}=\left|\Gamma_{1}(v) \cap \tilde{\mathrm{D}}_{r}^{1}\right|$ is a constant independent of $u, v, w$ by Lemma 3.5(iii). Hence $\gamma^{\prime}=\left|\Gamma_{2}(v) \cap \tilde{\mathrm{D}}_{r}^{1}\right|=\left|\tilde{\mathrm{D}}_{r}^{1}\right|-1-\alpha^{\prime}=a_{r}-1-\alpha^{\prime}$ is also a constant independent of $u, v, w$. Thus by Lemmas 3.8 and 3.10,

$$
\begin{aligned}
\langle(E \hat{u}-E \hat{w}), E \hat{v}\rangle & =|X|^{-1}\left(\theta_{1}-\theta_{r}\right), \\
\left\langle E \widetilde{\mathrm{D}}_{r}^{1}, E \hat{v}\right\rangle= & |X|^{-1}\left(\theta_{0}+\alpha^{\prime} \theta_{1}+\gamma^{\prime} \theta_{2}\right), \\
\left\langle E \widehat{\widetilde{\mathrm{D}}_{1}^{r}}, E \hat{v}\right\rangle= & |X|^{-1}\left(\left(c_{r}-\gamma\right) \theta_{r-1}+\left(a_{r}-\left(c_{r}-\gamma\right)-\left(b_{r}-\beta\right)\right) \theta_{r}\right. \\
& \left.+\left(b_{r}-\beta\right) \theta_{r+1}\right) .
\end{aligned}
$$

Hence $\left(\theta_{r}-\theta_{r+1}\right) \beta+\left(\theta_{r}-\theta_{r-1}\right) \gamma$ is a constant independent of $u, v, w$. Rewriting $\theta_{r}-\theta_{r+1}$, $\theta_{r}-\theta_{r-1}$ using Lemma 3.12 (here note that $t_{1} t_{0}^{-1}-t_{0} t_{1}^{-1} \neq 0$ by the assumption $t_{1} \notin$ $\left.\left\{t_{0},-t_{0}\right\}\right)$, and absorbing the common factors into the constant gives

$$
\left(\frac{t_{r+1}}{t_{r}}-\frac{t_{r}}{t_{r+1}}\right) \beta+\left(\frac{t_{r-1}}{t_{r}}-\frac{t_{r}}{t_{r-1}}\right) \gamma=\zeta_{2},
$$

a constant which is independent of $u, v, w$

The coefficient matrix for $\beta$ and $\gamma$ in the linear Eqs. (6), (8) is

$$
\left(\begin{array}{cc}
2-\frac{t_{r+1}}{t_{r}}-\frac{t_{r}}{t_{r+1}} & 2-\frac{t_{r}}{t_{r-1}}-\frac{t_{r-1}}{t_{r}} \\
\frac{t_{r+1}}{t_{r}}-\frac{t_{r}}{t_{r+1}} & \frac{t_{r-1}}{t_{r}}-\frac{t_{r}}{t_{r-1}}
\end{array}\right),
$$

which has determinant $2\left(t_{r+1}-t_{r}\right)\left(t_{r}-t_{r-1}\right)\left(t_{r+1}-t_{r-1}\right) /\left(t_{r-1} t_{r} t_{r+1}\right) \neq 0$. Thus $\beta, \gamma$ are independent of the choice of $u, v, w$. 


\section{Parameters}

In this section we prove a more complete version of Theorem 1.1, giving explicit formulas for the structure constants. We begin by recalling the following result.

Theorem 4.1 [12, Theorem 1.1] Let $\Gamma$ denote a distance-regular graph with diameter $d \geq 2$ and vertex set $X$. Let $W$ denote a spin model on $X$, and assume that $\Gamma$ supports $W$. Let $c_{i}, a_{i}, b_{i}(0 \leq i \leq d)$ denote the intersection numbers of $\Gamma$. Let $A_{0}, A_{1}, \ldots, A_{d}$ denote the distance matrices of $\Gamma$, write $W=\sum_{i=0}^{d} t_{i} A_{i}$, and set $x=t_{1} t_{0}^{-1}$ and $p=t_{0} t_{2} t_{1}^{-2}$. Then the following (i)-(iii) hold.

(i) $t_{i} t_{i-1}^{-1}=p^{i-1} x \quad(1 \leq i \leq d)$.

(ii) Suppose $a_{1}=0$. Then $a_{i}=0(1 \leq i<d)$. Moreover, if $p^{2} \neq 1$, then either $p^{d} x=1$ or $p^{d-1} x^{2}=-1$.

(iii) Suppose $x^{2} \neq 1$. Then the eigenvalues $\theta_{i}(0 \leq i \leq d)$ (in the standard ordering for $\Psi)$ and the intersection numbers $c_{i}(0<i<d), c_{d}, b_{0}$, and $b_{i}(0<i<d)$ of $\Gamma$ are given by

$$
\begin{aligned}
\theta_{i}= & \frac{p x^{2}-1}{x\left(p^{d-1} x+1\right)\left(1-p^{d} x^{2}\right)} \\
& \times\left(\left(p^{d+i-1} x^{3}+1\right)\left[\begin{array}{c}
d-i \\
1
\end{array}\right]_{p}+p^{d-i} x\left(p^{i-1} x+1\right)\left[\begin{array}{l}
i \\
1
\end{array}\right]_{p}\right), \\
c_{i}= & \frac{p^{i-1}(x-1)\left(p x^{2}-1\right)\left(p^{d-i} x+1\right)\left(p^{d+i-1} x^{2}-1\right)}{\left(p^{d-1} x+1\right)\left(p^{d} x^{2}-1\right)\left(p^{i-1} x-1\right)\left(p^{2 i-1} x^{2}-1\right)}\left[\begin{array}{l}
i \\
1
\end{array}\right]_{p} \\
c_{d}= & \frac{p^{d-1}\left(x^{2}-1\right)\left(p x^{2}-1\right)}{\left(p^{d} x^{2}-1\right)\left(p^{2 d-2} x^{2}-1\right)}\left[\begin{array}{l}
d \\
1
\end{array}\right]_{p} \\
b_{0}= & -\frac{\left(p x^{2}-1\right)\left(p^{d-1} x^{3}+1\right)}{x\left(p^{d-1} x+1\right)\left(p^{d} x^{2}-1\right)}\left[\begin{array}{l}
d \\
1
\end{array}\right]_{p} \\
b_{i}= & -\frac{p^{i}(x-1)\left(p x^{2}-1\right)\left(p^{i-1} x^{2}-1\right)\left(p^{d+i-1} x^{3}+1\right)}{x\left(p^{d-1} x+1\right)\left(p^{d} x^{2}-1\right)\left(p^{i} x-1\right)\left(p^{2 i-1} x^{2}-1\right)}\left[\begin{array}{c}
d-i \\
1
\end{array}\right]_{p},
\end{aligned}
$$

where

$$
\left[\begin{array}{l}
i \\
1
\end{array}\right]_{p}= \begin{cases}i & \text { if } p=1 \\
\frac{p^{i}-1}{p-1} & \text { otherwise }\end{cases}
$$

Moreover, all denominators are non-zero in these expressions.

We have omitted the formulas for the $a_{i}$, as they are determined by the well-known formula $a_{i}=b_{0}-b_{i}-c_{i}(0 \leq i \leq d)$.

Theorem 4.2 Let $\Gamma$ denote a distance-regular graph with diameter $d \geq 2$ and vertex set $X$. Suppose that $\Gamma$ supports a spin model with at least three distinct entries. Let $p, x$ be as in Theorem 4.1. Then one of the following holds: 
(i) For all $u \in X$, the subgraph induced on $\Gamma_{1}(u)$ is a disjoint union of cliques.

(ii) For all $u \in X$, the subgraph induced on $\Gamma_{1}(u)$ is a nontrivial strongly regular graph with parameters

$$
\begin{aligned}
v= & -\frac{\left(p x^{2}-1\right)\left(p^{d-1} x^{3}+1\right)}{x\left(p^{d-1} x+1\right)\left(p^{d} x^{2}-1\right)}\left[\begin{array}{l}
d \\
1
\end{array}\right]_{p}, \\
k= & -\frac{\left(p x^{2}-1\right)\left(p^{d} x-1\right)(x+1)\left(p^{d-1} x^{2}+1\right)}{x\left(p^{d-1} x+1\right)(p x-1)\left(p^{d} x^{2}-1\right)}, \\
\lambda= & \frac{\left(p x^{2}-1\right)}{x(p x-1)^{2}\left(p^{d-1} x+1\right)\left(p^{d} x^{2}-1\right)} \\
& \times\left(p x(x+2)\left(-p^{2 d-1} x^{3}+p^{d-1} x-p^{d-2} x+1\right)\right. \\
& \left.+(2 x+1)\left(p^{2 d-1} x^{3}-p^{d+1} x^{2}+p^{d} x^{2}-1\right)\right), \\
\mu= & \frac{(p-1)\left(p x^{2}-1\right)\left(p^{d-1} x^{2}+1\right)\left(p^{d} x-1\right)}{(p x-1)^{2}\left(p^{d-1} x+1\right)\left(p^{d} x^{2}-1\right)} .
\end{aligned}
$$

Moreover, case (i) occurs if and only if $p=1$ or $a_{i}=0(0 \leq i \leq d-1)$.

Proof: Let $\Delta$ denote the subgraph induced on $\Gamma_{1}(u)$. Then by the distance-regularity of $\Gamma$, $\Delta$ contains $v=b_{0}$ many vertices and every vertex of $\Delta$ has exactly $k=a_{1}$ many neighbors in $\Delta$.

Pick $v, w \in \Delta$ which are adjacent in $\Delta$. Then the number of common neighbors of $v$ and $w$ in $\Delta$ is $\lambda=e\left(w, \mathrm{D}_{1}^{1}(u, v)\right)$, which is independent of $u, v, w$ by Lemma 3.5(iii). Now pick $v^{\prime}, w^{\prime} \in \Delta$ which have distance two in $\Gamma$. Then they have $\mu=e\left(w^{\prime}, \mathrm{D}_{1}^{1}\left(u, v^{\prime}\right)\right)$ common neighbors in $\Delta$, which is independent of $u, v^{\prime}, w^{\prime}$ by Lemma 3.5(iii). If $\mu=$ 0 , then $\Delta$ is a disjoint union of cliques. If $\mu>0$, then $\Delta$ is connected with diameter two.

The formulas are computed by substituting the formulas of Theorem 4.1 into the equations for $e\left(w, \mathrm{D}_{1}^{1}(u, v)\right)$ and $e\left(w^{\prime}, \mathrm{D}_{1}^{1}\left(u, v^{\prime}\right)\right)$ which appear in the proof of Lemma 3.5(iii). This does not depend upon whether or not $\mu=0$. Examining the formula for $\mu$, we find that $\mu=0$ if and only if one of the factors $p-1, p^{d} x-1$, or $p^{d-1} x^{2}+1$ is zero. In particular, either every first subconstituent is a disjoint union of $b_{0} /\left(a_{1}+1\right)$ many cliques of size $a_{1}+1$, or none is a disjoint union of cliques. If one of $p^{d} x-1=0$ or $p^{d-1} x^{2}+1=0$, then $a_{i}=0$ $(0 \leq i \leq d-1)$ by Theorem 4.1(ii).

It follows that a distance-regular graph with $a_{1}=0$ which supports a spin model is 2homogeneous and either bipartite or almost bipartite. The graphs with these combinatorial properties are characterized by their parameters in $[34,36]$.

Lemma 4.3 Let $\Gamma$ denote a distance-regular graph with diameter $d \geq 2$, and suppose that $\Gamma$ supports a spin model with at least three distinct entries. Let $p, x$ be as in Theorem 4.1 . If $p=1$, then $\Gamma$ is a Hamming graph. 
Proof: By Theorem 4.2, $\mathrm{D}_{1}^{1}(u, v)$ is a clique for every edge $(u, v)$ of $\Gamma$. Furthermore, $c_{i}=i, a_{i}=i(q-2)(0 \leq i \leq d)$ by [12, Lemma 5.2], where $q=-x^{-1}(x-1)^{2}$. Now [16] implies that $\Gamma$ is the Hamming graph $H(d, q)$.

Theorem 4.4 Let $\Gamma$ denote a distance-regular graph with diameter $d \geq 2$, and suppose that $\Gamma$ supports a spin model with at least three distinct entries. Let $p, x$ be as in Theorem 4.1. Pick any vertex $u$. Then the subgraph induced on $\Gamma_{d}(u)$ is distance-regular with diameter $d^{\prime}$ and intersection numbers $c_{i}^{\prime}\left(1 \leq i \leq d^{\prime}\right), a_{i}^{\prime}\left(0 \leq i \leq d^{\prime}\right)$, and $b_{i}^{\prime}\left(0 \leq i \leq d^{\prime}-1\right)$ given by

$$
\begin{aligned}
c_{i}^{\prime} & =\frac{p^{i-1}(x-1)\left(p x^{2}-1\right)\left(p^{2 d-i-1} x^{2}-1\right)\left(p^{d+i-1} x^{2}-1\right)}{\left(p^{2 d-2} x^{2}-1\right)\left(p^{i-1} x-1\right)\left(p^{d} x^{2}-1\right)\left(p^{2 i-1} x^{2}-1\right)}\left[\begin{array}{l}
i \\
1
\end{array}\right]_{p}, \\
a_{i}^{\prime} & =-\frac{\left(p^{d} x-1\right)\left(p x^{2}-1\right)\left(p^{i-1} x^{2}-1\right)\left(p^{2 d-2} x^{3}-1\right)}{x\left(p^{2 d-2} x^{2}-1\right)\left(p^{i-1} x-1\right)\left(p^{i} x-1\right)\left(p^{d} x^{2}-1\right)}\left[\begin{array}{c}
i \\
1
\end{array}\right]_{p}, \\
b_{i}^{\prime} & =-\frac{p^{i}(x-1)\left(p x^{2}-1\right)\left(p^{i-1} x^{2}-1\right)\left(p^{2 d+i-2} x^{4}-1\right)}{x\left(p^{2 d-2} x^{2}-1\right)\left(p^{i} x-1\right)\left(p^{d} x^{2}-1\right)\left(p^{2 i-1} x^{2}-1\right)}\left[\begin{array}{c}
d-i \\
1
\end{array}\right]_{p}, \\
d^{\prime} & =\min \left(\{d\} \cup\left\{i \mid i \geq 0, p^{2 d+i-2} x^{4}-1=0\right\}\right) .
\end{aligned}
$$

Proof: Pick any $v \in \Gamma_{d}(u)$, and set $\mathrm{D}_{j}^{i}=\mathrm{D}_{j}^{i}(u, v)$. Observe that $\Gamma_{d}(u)=\bigcup_{i=0}^{d} \mathrm{D}_{i}^{d}$. Suppose $\mathrm{D}_{i}^{d} \neq \emptyset$ for some $i$, and pick any $w \in \mathrm{D}_{i}^{d}$. Then by Lemma 3.5(iv), the numbers $e\left(w, \mathrm{D}_{i-1}^{d}\right), e\left(w, \mathrm{D}_{i}^{d}\right)$, and $e\left(w, \mathrm{D}_{i+1}^{d}\right)$ are expressed in terms of the parameters $\theta_{r}, c_{r}, a_{r}$, $b_{r}(0 \leq i \leq d)$. Substitute the formulas of Theorem 4.1 into these expressions to get formulas for $e\left(w, \mathrm{D}_{i-1}^{d}\right), e\left(w, \mathrm{D}_{i}^{d}\right)$, and $e\left(w, \mathrm{D}_{i+1}^{d}\right)$ in terms of $p$ and $x$, and observe that these formulas coincide with the formulas for $c_{i}^{\prime}, a_{i}^{\prime}, b_{i}^{\prime}$ in the statement of the theorem.

Observe that every factor in the numerator of the formula for $e\left(w, \mathrm{D}_{i-1}^{d}\right)$ also appears as a factor of either $c_{i}$ or $c_{d-i}$ in Theorem 4.1. Since $c_{i} \neq 0(1 \leq i \leq d)$, this implies $e\left(w, \mathrm{D}_{i-1}^{d}\right) \neq 0$ for $w \in \mathrm{D}_{i}^{d}(1 \leq i \leq d)$. This means that there is a path of length $i$ in $\Gamma_{d}(u)$ between $v$ and $w$. Hence the subgraph $\Delta$ induced on $\Gamma_{d}(u)$ is connected, and every pair of vertices in $\Delta$ have the same distance in $\Delta$ as in $\Gamma$. Now it is easy to see that $\Delta$ is a distance-regular graph with the intersection numbers $c_{i}^{\prime}, a_{i}^{\prime}, b_{i}^{\prime}$ given in the statement of the theorem.

Observe that the formula for $b_{i}^{\prime}$ is zero when $p^{2 d+i-2} x^{4}-1=0$, so the diameter cannot exceed the least nonnegative integer $i$ satisfying this condition. For any smaller $i<d^{\prime}$ the expression for $b_{i}^{\prime}$ is nonzero, so the diameter is at least this large. Of course $d^{\prime} \leq d$.

Remark In all known examples of distance-regular graphs which support a spin model, the induced graph on $\Gamma_{d}(u)$ has diameter 0,1 , or $d$. If $p^{d-1} x^{2}+1=0$ (ie. $\Gamma$ is bipartite), then $d^{\prime}=0$. If $p^{d} x-1=0$ (ie. $\Gamma$ is almost bipartite), then either $d^{\prime}=d$ or $d^{\prime}=1$. If $p=1$ (i.e. $\Gamma$ is a Hamming graph), then $d^{\prime}=d$ unless $\Gamma$ is a Hamming cube, in which case $d^{\prime}=0$.

Suppose $\Gamma$ is 1-homogeneous. Fix adjacent vertices $u, v$ and write $\mathrm{D}_{j}^{i}=\mathrm{D}_{j}^{i}(u, v)$. Define $\alpha_{r}(1 \leq r \leq d-1)$ as follows: For any $w \in \mathrm{D}_{r}^{r+1}, \alpha_{r}=e\left(w, \mathrm{D}_{r}^{r+1}\right)$. Define $\gamma_{r}, \beta_{r}$ $(1 \leq r \leq d-1)$ as follows: For any $w^{\prime} \in \mathrm{D}_{r}^{r}, \gamma_{r}=e\left(w^{\prime}, \mathrm{D}_{r-1}^{r-1}\right), \beta_{r}=e\left(w^{\prime}, \mathrm{D}_{r+1}^{r+1}\right)$. From 
these three structure constants, all others are readily computed using Lemma 3.2, as in the proofs of Lemmas 3.5(ii) and 3.13.

Theorem 4.5 Let $\Gamma$ denote a distance-regular graph with diameter $\geq 2$, and suppose that $\Gamma$ supports a spin model with at least three distinct entries. Then $\Gamma$ is 1-homogeneous with parameters $\alpha_{r}(0 \leq r \leq d-1), \gamma_{r}(1 \leq r \leq d-1), \beta_{r}(1 \leq r \leq d-1)$ given by

$$
\begin{aligned}
\alpha_{r} & =-\frac{\left(p x^{2}-1\right)\left(p^{d} x-1\right)\left(p^{d-1} x^{2}+1\right)\left(p^{r} x^{2}-1\right)}{x\left(p^{d-1} x+1\right)\left(p^{d} x^{2}-1\right)\left(p^{r} x-1\right)^{2}}\left[\begin{array}{c}
r \\
1
\end{array}\right]_{p} \\
\beta_{r} & =-\frac{p^{r}(x-1)\left(p x^{2}-1\right)\left(p^{r-1} x-1\right)\left(p^{r} x^{2}-1\right)\left(p^{d+r-1} x^{3}+1\right)}{x\left(p^{d-1} x+1\right)\left(p^{r} x-1\right)^{2}\left(p^{d} x^{2}-1\right)\left(p^{2 r-1} x^{2}-1\right)}\left[\begin{array}{c}
d-r \\
1
\end{array}\right]_{p}, \\
\gamma_{r} & =\frac{p^{r-1}(x-1)\left(p x^{2}-1\right)\left(p^{r} x-1\right)\left(p^{d-r} x+1\right)\left(p^{d+r-1} x^{2}-1\right)}{\left(p^{d-1} x+1\right)\left(p^{d} x^{2}-1\right)\left(p^{r-1} x-1\right)^{2}\left(p^{2 r-1} x^{2}-1\right)}\left[\begin{array}{c}
r-1 \\
1
\end{array}\right]_{p} .
\end{aligned}
$$

Proof: Let $u, v, w \in X$ be vertices with $\partial(u, v)=1, \partial(u, w)=r$, and $\partial(v, w)=s$. Set $\mathrm{D}_{j}^{i}=\mathrm{D}_{j}^{i}(u, v)$. Then $e\left(w, \mathrm{D}_{j}^{i}\right)$ is independent of $u, v, w$ for all $i$ and $j$ by Lemma 3.5(ii), (iii), (iv) and Lemma 3.13. Thus $\Gamma$ is 1-homogeneous.

Observe that for $1 \leq r \leq d-1$ the number $\alpha_{r}$ is the number $\alpha$ appearing in the proof of Lemma 3.5(ii). Solve for $\alpha$ with the formulas of Theorem 4.1 to compute $\alpha_{r}$.

We use the fact that $\Gamma$ is 1-homogeneous to compute $\beta_{r}$ and $\gamma_{r}(1 \leq r \leq d-1)$ from $\alpha_{r}$. We claim that $a_{r} \beta_{r}=b_{r} \alpha_{r}$. This formula is obtained by counting the number of edges between the sets $\mathrm{D}_{r}^{r}$ and $\mathrm{D}_{r}^{r+1}$ in two ways. Each of the vertices in $\mathrm{D}_{r}^{r}$ has $b_{r}-\beta_{r}$ many neighbors in $\mathrm{D}_{r}^{r+1}$, while each of the vertices in $\mathrm{D}_{r}^{r+1}$ has $a_{r}-\alpha_{r}$ many neighbors in $\mathrm{D}_{r}^{r}$. Furthermore, $\left|\mathrm{D}_{r}^{r}\right|=p_{r, r}^{1}=k_{r} a_{r} / k_{1}$ and $\left|\mathrm{D}_{r}^{r+1}\right|=p_{r+1, r}^{1}=k_{r} b_{r} / k_{1}$ by the definition of $p_{r, r}^{1}, p_{r+1, r}^{1}$ and (1). The claim follows. The computation of $\beta_{r}$ from $\alpha_{r}$ is now straight forward.

A similar argument gives $\gamma_{r}$. Here we find that $a_{r} \gamma_{r}=c_{r} \alpha_{r-1}$ by counting the number of edges between the sets $\mathrm{D}_{r}^{r}$ and $\mathrm{D}_{r-1}^{r}$ in two ways. Each of the vertices in $\mathrm{D}_{r}^{r}$ has $c_{r}-\gamma_{r}$ many neighbors in $\mathrm{D}_{r-1}^{r}$, while each of the vertices in $\mathrm{D}_{r-1}^{r}$ has $a_{r}-\alpha_{r-1}$ neighbors in $\mathrm{D}_{r}^{r}$. Furthermore, $\left|\mathrm{D}_{r}^{r}\right|=k_{r} a_{r} / k_{1}$ and $\left|\mathrm{D}_{r-1}^{r}\right|=p_{r, r-1}^{1}=k_{r} c_{r} / k_{1}$ by the definition of $p_{r, r-1}^{1}$ and (1).

\section{References}

1. E. Bannai, Et. Bannai, T. Ikuta, and K. Kawagoe, "Spin models constructed from the Hamming association schemes," in Proceedings of the 10th Algebraic Combinatorics Symposium at Gifu University, 1992.

2. E. Bannai and Et. Bannai, "Spin models on finite cyclic groups," J. Algebraic Combin. 3 (1994), 243-259.

3. E. Bannai and Et. Bannai, "Generalized generalized spin models (four-weight spin models)," Pacific J. Math. 170 (1995), 1-16.

4. E. Bannai, Et. Bannai, and F. Jaeger, "On spin models, modular invariance, and duality,” J. Alg. Combin. 6 (1997), 203-228.

5. E. Bannai and T. Ito, Algebraic Combinatorics I, Benjamin/Cummings, Menlo Park, 1984.

6. A.E. Brouwer, A.M. Cohen, and A. Neumaier, Distance-Regular Graphs, Springer, New York, 1989.

7. P.J. Cameron, J.M. Goethals, and J.J. Seidel, "Strongly regular graphs having strongly regular subconstituents," J. Alg. 55 (1978), 257-280. 
8. J.S. Caughman, "The last subconstituent of a bipartite P- and Q-polynomial association scheme," European J. Combin. 24(5) (2003), 459-470.

9. B. Curtin, "2-homogeneous bipartite distance-regular graphs," Discrete Math. 187 (1998), 39-70.

10. B. Curtin, "Distance-regular graphs which support a spin model are thin," Discrete Math. 197/198 (1999), 205-216.

11. B. Curtin, "The Terwilliger algebra of a 2-homogeneous bipartite distance-regular graph," J. Combin. Theory Ser. B 81 (2001), 125-141.

12. B. Curtin and K. Nomura, "Some formulas for spin models on distance-regular graphs," J. Combin. Theory Ser. B 75 (1999), 206-236.

13. B. Curtin and K. Nomura, "Distance-regular graphs related to the quantum enveloping algebra of $s l(2)$," J. Algebraic Combin. 12 (2000), 25-36.

14. B. Curtin and K. Nomura, "Spin models and hyper-self-dual Bose-Mesner algebras," J. Alg. Combin. 13 (2001), 173-186

15. P. Delsarte, "An algebraic approach to the association schemes of coding theory," Philips Research Reports Supplements 10 (1973).

16. Y. Egawa, "Characterization of $H(n, q)$ by the parameters," J. Combin. Theroy Ser. A 31 (1981), 108-125.

17. P. de la Harpe, "Spin models for link polynomials, strongly regular graphs and Jaeger's Higman-Sims model," Pacific J. Math. 162 (1994), 57-96.

18. F. Jaeger, "Towards a classification of spin models in terms of association schemes," Advanced Studies in Pure Math. 24 (1996), 197-225.

19. F. Jaeger, "Strongly regular graphs and spin models for the Kauffman polynomial," Geom. Dedicata 44 (1992), 23-52.

20. F. Jaeger, "On spin models, triply regular association schemes, and duality," J. Alg. Combin. 4 (1995), 103-144.

21. F. Jaeger, M. Matsumoto, and K. Nomura, "Bose-Mesner algebras related to type II matrices and spin models," J. Alg. Combin. 8 (1998), 39-72.

22. V.F.R. Jones, "On knot invariants related to some statistical mechanical models," Pac. J. Math. 137 (1989), 311-224.

23. A. Jurisic, "AT4 family and 2-homogeneous graphs," The 2000 COM 2 Mac Conference on Association Schemes, Codes and Designs (Pohang). Discrete Math. 264(1-3) (2003), 127-148.

24. A. Jurisic and J. Koolen, "Krein parameters and antipodal tight graphs with diameter 3 and 4," Discrete Math. 244 (2002), 181-202.

25. A. Jurisic and J. Koolen, "1-homogeneous graphs with Cocktail party $\mu$-graphs.” (English. English Summary) J. Algebraic Combin. 18(2) (2003), 79-98. 05C12 (05E30).

26. A. Jurisic and J. Koolen, "A local approach to 1-homogeneous graphs," Designs, Codes and Cryptography 21 (2000), 127-147.

27. A. Jurisic, J. Koolen, and P. Terwilliger, "Tight distance-regular graphs with small diameter," preprint.

28. A. Jurisic, J. Koolen, and P. Terwilliger, "Tight distance-regular graphs," J. Algebraic Combin. 12 (2000), $163-197$.

29. A. Kasikova, "Distance-regular graphs with strongly regular subconstituents," J. Algebraic Combin. 6 (1997), 247-252.

30. K. Kawagoe, A. Munemasa, and Y. Watatani, "Generalized spin models," J. Knot Th. Ramif. 3 (1995), 465-475.

31. A. Neumaier, "Duality in coherent configurations," Combinatorica 9 (1989), 59-67.

32. K. Nomura, "Homogeneous graphs and regular near polygons," J. Combin. Theory Ser. B 60 (1994) 63-71.

33. K. Nomura, "Spin models constructed from Hadamard matrices," J. Combin. Theory Ser. A 68 (1994), 251-261.

34. K. Nomura, "Spin models on bipartite distance-regular graphs," J. Combin. Theory Ser. B 64 (1995), 300-313.

35. K. Nomura, "Spin models on triangle-free connected graphs," J. Combin. Theory Ser. B 67 (1996), 284-295.

36. K. Nomura, "Spin models and almost bipartite 2-homogeneous graphs," Advanced Studies in Pure Math. 24 (1996), 285-308

37. K. Nomura, "An algebra associated with a spin model," J. Alg. Combin. 6 (1997), 53-58.

38. P. Terwilliger, “A new inequality for distance-regular graphs," Discrete Math. 137 (1995), 319-332.

39. N. Yamazaki, "Bipartite distance-regular graphs with an eigenvalue of multiplicity $k$," J. Combin. Theory Ser. B 66 (1995), 34-37. 